\title{
Symmetry Reductions, Exact Solutions, and Conservation Laws of a Modified Hunter-Saxton Equation
}

\author{
Andrew Gratien Johnpillai ${ }^{1}$ and Chaudry Masood Khalique ${ }^{2}$ \\ ${ }^{1}$ Department of Mathematics, Eastern University, Sri Lanka \\ ${ }^{2}$ International Institute for Symmetry Analysis and Mathematical Modelling, Department of Mathematical Sciences, \\ North-West University, Mafikeng Campus, Private Bag X 2046, Mmabatho 2735, South Africa
}

Correspondence should be addressed to Chaudry Masood Khalique; masood.khalique@nwu.ac.za

Received 29 June 2013; Accepted 14 August 2013

Academic Editor: Maria Gandarias

Copyright (C) 2013 A. G. Johnpillai and C. M. Khalique. This is an open access article distributed under the Creative Commons Attribution License, which permits unrestricted use, distribution, and reproduction in any medium, provided the original work is properly cited.

We study a modified Hunter-Saxton equation from the Lie group-theoretic point of view. The Lie point symmetry generators of the underlying equation are derived. We utilize the Lie algebra admitted by the equation to obtain the optimal system of onedimensional subalgebras of the Lie algebra of the equation. These subalgebras are then used to reduce the underlying equation to nonlinear third-order ordinary differential equations. Exact traveling wave group-invariant solutions for the equation are constructed by integrating the reduced ordinary differential equations. Moreover, using the variational method, we construct infinite number of nonlocal conservation laws by the transformation of the dependent variable of the underlying equation.

\section{Introduction}

The nonlinear partial differential equation

$$
\left(u_{t}+u u_{x}\right)_{x x}=\frac{1}{2}\left(u_{x}^{2}\right)_{x}
$$

was proposed by Hunter and Saxton [1]. They showed that the weakly nonlinear waves are described by (1), where $u(x, t)$ describes the director field of a nematic liquid crystal, $x$ is a space variable in a reference frame moving with the linearized wave velocity, and $t$ is a slow time variable. Liquid crystals are fluids made up of long rigid molecules. Equation (1) is a high-frequency limit of the CamassaHolm equation [2]. Hunter and Zheng [3] showed that it is bivariational, bi-Hamiltonian, and a member of the Harry Dym hierarchy of integrable flows. It is well known that (1) under reciprocal transformation reduces to the Liouville equation [2]. Also, an interesting geometric interpretation of the Hunter-Saxton equation $[4,5]$ is that, for spatially periodic functions, it describes the geodesic flow on the homogeneous space $\operatorname{Vir}(\mathbb{S}) / \operatorname{Rot}(S)$ of the Virasoro group $\operatorname{Vir}(S)$ modulo the rotations $\operatorname{Rot}(S)$, with respect to the rightinvariant homogeneous $\dot{H}^{1}$ metric: $\langle f, g\rangle=\int_{S} f_{x} g_{x} d x$.
In this paper the modified Hunter-Saxton equation [6]

$$
u_{t}-2 u_{x} u_{x x}-u u_{x x x}-u_{x x t}=0
$$

is studied from the Lie group analysis standpoint. We first obtain the Lie point symmetry generators and then utilize the Lie algebra admitted by the equation to obtain the optimal system of one-dimensional subalgebras of the Lie algebra of the equation. These subalgebras are later used to reduce the modified Hunter-Saxton equation to nonlinear third-order ordinary differential equations. We then construct exact traveling wave group-invariant solutions for the equation by integrating the reduced ordinary differential equations. Finally, using the variational method, we construct infinite number of nonlocal conservation laws by the transformation of the dependent variable of the underlying equation.

The outline of the paper is as follows. In Section 2, we briefly discuss some main operator identities and their relationship. In Section 3, we present the Lie point symmetry generators of (2). In Section 4, the optimal system of onedimensional subalgebras of the Lie symmetry algebra of (2) is constructed. Moreover, using the optimal system of subalgebras, symmetry reductions and exact group-invariant 
solutions of (2) are obtained. In Section 5, nonlocal conservation laws of (2) are constructed using Noether's theorem. Finally, in Section 6, concluding remarks are presented.

\section{Preliminaries}

In this section we present the notations that will be used in the sequel. For details, the reader is referred to $[7,8]$.

Consider a $k$ th-order system of partial differential equations (PDEs) of $n$ independent variables $x=\left(x^{1}, x^{2}, \ldots, x^{n}\right)$ and $m$ dependent variables $u=\left(u^{1}, u^{2}, \ldots, u^{m}\right)$; namely,

$$
E^{\alpha}\left(x, u, u_{(1)}, \ldots, u_{(k)}\right)=0, \quad \alpha=1, \ldots, m,
$$

where $u_{(1)}, u_{(2)}, \ldots, u_{(k)}$ denote the collections of all first, second,..., $k$ th-order partial derivatives; that is, $u_{i}^{\alpha}=D_{i}\left(u^{\alpha}\right)$, $u_{i j}^{\alpha}=D_{j} D_{i}\left(u^{\alpha}\right), \ldots$, respectively, with the total differentiation operator with respect to $x^{i}$ being defined by

$$
D_{i}=\partial_{x^{i}}+u_{i}^{\alpha} \partial_{u^{\alpha}}+u_{i j}^{\alpha} \partial_{u_{j}^{\alpha}}+\cdots, \quad i=1, \ldots, n,
$$

and the summation convention is used whenever appropriate.

The conserved vector $T=\left(T^{1}, T^{2}, \ldots, T^{n}\right)$ of $(3)$, where each $T^{i} \in \mathscr{A}, \mathscr{A}$ is the space of all differential functions, satisfies the equation

$$
D_{i} T^{i}=0
$$

along the solutions of (3).

The Lie point symmetry generator in the form of infinite formal sum is given by

$$
X=\xi^{i} \partial_{x^{i}}+\eta^{\alpha} \partial_{u^{\alpha}}+\sum_{s \geq 1} \zeta_{i_{1} i_{2} \cdots i_{s}}^{\alpha} \partial_{u_{i_{1} i_{2} \cdots i_{s}}^{\alpha}},
$$

where $\xi$ and $\eta$ are functions of $x$ and $u$ and are independent of derivatives of $u$, and the additional coefficients are determined uniquely by the prolongation formulae

$$
\begin{gathered}
\zeta_{i}^{\alpha}=D_{i}\left(W^{\alpha}\right)+\xi^{j} u_{i j}^{\alpha}, \\
\zeta_{i_{1} \cdots i_{s}}^{\alpha}=D_{i_{1}} \cdots D_{i_{s}}\left(W^{\alpha}\right)+\xi^{j} u_{j i_{1} \cdots i_{s}}^{\alpha}, \quad s>1,
\end{gathered}
$$

in which $W^{\alpha}$ is the Lie characteristic function defined by $W^{\alpha}=\eta^{\alpha}-\xi^{j} u_{j}^{\alpha}$.

Let $L=L\left(x, u, u_{(1)}, \ldots, u_{(l)}\right) \in \mathscr{A}, l \leq k$ be a Lagrangian associated with a Noether symmetry operator $X$. If $B^{1}, \ldots, B^{n}$ are point-dependent gauge terms, then the Noether symmetry operator $X$ is determined by

$$
X L+L D_{i} \xi^{i}=D_{i} B^{i}, \quad i=1, \ldots, n,
$$

and the conserved vectors, $T^{i}$, corresponding to each $X$

$$
\begin{aligned}
T^{i}= & B^{i}-L \xi^{i}-W^{\alpha} \frac{\partial L}{\partial u_{i}^{\alpha}} \\
& -\left[D_{j}\left(W^{\alpha}\right)-W^{\alpha} D_{j}\right] \frac{\partial L}{\partial u_{i j}^{\alpha}}, \quad i=1, \ldots, n
\end{aligned}
$$

\section{Lie Point Symmetries of (2)}

In this section, we discuss in brief the Lie symmetry group method to obtain Lie point symmetry generators admitted by (2). For detailed account of this method see [10-14] and the references therein.

A vector field

$$
X=\tau(t, x, u) \partial_{t}+\xi(t, x, u) \partial_{x}+\eta(t, x, u) \partial u
$$

is a generator of point symmetry of (2), if

$$
\left.X^{[3]}\left(u_{t}-2 u_{x} u_{x x}-u u_{x x x}-u_{x x t}\right)\right|_{(2)}=0
$$

where the operator $X^{[3]}$ is the third prolongation of the operator $X$ defined by

$$
X^{[3]}=X+\zeta_{t} \partial_{u_{t}}+\zeta_{x} \partial_{u_{x}}+\zeta_{x x x} \partial_{u_{x x x}}+\zeta_{x x t} \partial_{u_{x x t}}
$$

with the coefficients $\zeta_{t}, \zeta_{x}, \zeta_{x x x}$, and $\zeta_{x x t}$ being given by

$$
\begin{gathered}
\zeta_{t}=D_{t}(\eta)-u_{t} D_{t}(\tau)-u_{x} D_{t}(\xi), \\
\zeta_{x}=D_{x}(\eta)-u_{t} D_{x}(\tau)-u_{x} D_{x}(\xi), \\
\zeta_{x x}=D_{x}\left(\zeta_{x}\right)-u_{x t} D_{x}(\tau)-u_{x x} D_{x}(\xi), \\
\zeta_{x x x}=D_{x}\left(\zeta_{x x}\right)-u_{x x t} D_{x}(\tau)-u_{x x x} D_{x}(\xi), \\
\zeta_{x x t}=D_{t}\left(\zeta_{x x}\right)-u_{x x t} D_{t}(\tau)-u_{x x x} D_{t}(\xi) .
\end{gathered}
$$

Here, $D_{t}$ and $D_{x}$ are the total derivative operators defined by

$$
\begin{aligned}
& D_{t}=\partial_{t}+u_{t} \partial_{u}+\cdots \\
& D_{x}=\partial_{x}+u_{x} \partial_{u}+\cdots
\end{aligned}
$$

The coefficient functions $\tau$, $\xi$, and $\eta$ are independent of the derivatives of $u$; thus equating the coefficients of like derivatives of $u$ in the determining equation (11) yields the following over determined system of linear PDEs:

$$
\begin{gathered}
\tau=\tau(t), \quad \xi=\xi(t, x), \quad \eta_{u u}=0, \\
\xi_{x x}-2 \eta_{x u}=0, \quad 2 \xi_{x}-\eta_{x x u}=0, \\
\tau_{t}-3 \xi_{x}+\eta_{u}+\eta_{x x u}=0, \\
\eta_{t}-\eta_{t x x}-u \eta_{x x x}=0, \\
\xi_{t}-u \tau_{t}+3 u \xi_{x}-u \eta_{x x u}-\eta=0, \\
2 \xi_{t x}+3 u \xi_{x x}-2 \eta_{x}-\eta_{t u}-3 u \eta_{x u}=0, \\
\xi_{t x x}+u \xi_{x x x}-\xi_{t}-2 \eta_{x x}-2 \eta_{t x u}-3 u \eta_{x x u}=0 .
\end{gathered}
$$

Solving the determining equations (15) for $\tau, \xi$, and $\eta$, we obtain the three-dimensional Lie algebra spanned by the following Lie point symmetry generators admitted by (2):

$$
X_{1}=\partial_{t}, \quad X_{2}=\partial_{x}, \quad X_{3}=t \partial_{t}-u \partial_{u} .
$$


TABLE 1: Commutator table of the Lie algebra of (2).

\begin{tabular}{cccc}
\hline & $X_{1}$ & $X_{2}$ & $X_{3}$ \\
\hline$X_{1}$ & 0 & 0 & $X_{1}$ \\
$X_{2}$ & 0 & 0 & 0 \\
$X_{3}$ & $-X_{1}$ & 0 & 0 \\
\hline
\end{tabular}

TABLE 2: Adjoint table of the Lie algebra of (2).

\begin{tabular}{cccc}
\hline Ad & $X_{1}$ & $X_{2}$ & $X_{3}$ \\
\hline$X_{1}$ & $X_{1}$ & $X_{2}$ & $X_{3}-\epsilon X_{1}$ \\
$X_{2}$ & $X_{1}$ & $X_{2}$ & $X_{3}$ \\
$X_{3}$ & $e^{\epsilon} X_{1}$ & $X_{2}$ & $X_{3}$ \\
\hline
\end{tabular}

TABLE 3: Subalgebras, group invariants, and group-invariant solutions of (2).

\begin{tabular}{cccc}
\hline$N$ & $X$ & $\gamma$ & Group-invariant solution \\
\hline 1 & $X_{2}$ & $t$ & $u=h(\gamma)$ \\
2 & $X_{1}+\epsilon X_{2}$ & $x-\epsilon t$ & $u=h(\gamma)$ \\
3 & $X_{3}+a X_{2}$ & $x-a \ln t$ & $u=(1 / t) h(\gamma)$ \\
\hline
\end{tabular}

Here $\epsilon=0, \pm 1$, and $a$ is an arbitrary constant.

\section{Symmetry Reductions and Group-Invariant Solutions of $(2)$}

Here we utilize the Lie point symmetry generators (16) of (2) found in Section 3 to obtain symmetry reduction and construct exact group-invariant solutions for (2).

We first present the optimal system of one-dimensional subalgebras of the Lie algebra admitted by (2). The onedimensional subalgebras are then used to reduce (2) to ordinary differential equations (ODEs). Exact group-invariant solutions for the underlying equation (2) are constructed by integrating the reduced ODEs.

The results on the classification of the Lie point symmetries of (2) are summarized in the Tables 1, 2, and 3. The commutator table of the Lie point symmetries of (2) and the adjoint representations of the symmetry group of (2) on its Lie algebra are given in Tables 1 and 2, respectively. Tables 1 and 2 are then used to construct the optimal system of onedimensional subalgebras for (2) which are given in Table 3 (for more details of the approach see [10]).

Case 1. In this case, the group-invariant solution corresponding to the symmetry generator $X_{2}$ leads to the trivial solution $u(x, t)=C$, where $C$ is a constant.

Case 2. The group-invariant solution arising from the subalgebra $X_{1}+\epsilon X_{2}$ reduces (2) to the following nonlinear thirdorder ODE:

$$
(h-\epsilon) h^{\prime \prime \prime}+2 h^{\prime} h^{\prime \prime}+\epsilon h^{\prime}=0, \quad \epsilon \neq 0,
$$

where "prime" denotes differentiation with respect to $\gamma$. Integrating (17) twice with respect to $\gamma$, we obtain

$$
h^{\prime 2}=\frac{\epsilon h^{2}+K_{1} h+K_{2}}{\epsilon-h},
$$

where $K_{1}$ and $K_{2}$ are arbitrary constants of integration. Separating the variables in (18), integrating, and reverting back to our original variables, one obtains the solution of (2) in quadrature

$$
\int \sqrt{\frac{\epsilon-u}{\epsilon u^{2}+K_{1} u+K_{2}}} d u=K_{3} \pm(x-\epsilon t)
$$

where $K_{3}$ is an arbitrary constant of integration. One can obtain two particular solutions for (17) given by

$$
\begin{gathered}
h(\gamma)=\frac{1}{B}\left(\delta \pm \frac{B \gamma}{2}\right)^{2}-\frac{A}{B}, \\
h(\gamma)=-\frac{\epsilon}{4}(\delta \pm \gamma)^{2},
\end{gathered}
$$

where $A=C_{1}-\epsilon^{2}, B=-\epsilon$, and $C_{1}, \delta$ are arbitrary constants. Hence, we obtain the two special group-invariant solutions for (2) given by

$$
\begin{gathered}
u(x, t)=-\frac{1}{\epsilon}\left[\left(\delta \pm \frac{\epsilon(\epsilon t-x)}{2}\right)^{2}-\left(C_{1}-\epsilon^{2}\right)\right] \\
u(x, t)=-\frac{\epsilon}{4}[\delta \pm(x-\epsilon t)]^{2} .
\end{gathered}
$$

Likewise, one can obtain the following three group-invariant steady state solutions of (2) for the case when $\epsilon=0$ :

$$
\begin{gathered}
u(x, t)=\sqrt{C}_{1}\left(C_{3} \pm x\right), \\
u(x, t)=\left\{\frac{9 C_{2}}{4}\left(C_{3} \pm x\right)^{2}\right\}^{1 / 3}, \\
\sqrt{C_{1} u\left(C_{1} u+C_{2}\right)}-C_{2} \log \left(\sqrt{C_{1}\left(C_{1} u+C_{2}\right)}+C_{1} \sqrt{u}\right) \\
=C_{1}^{3 / 2}\left(C_{3} \pm x\right) .
\end{gathered}
$$

Here, $C_{1}, C_{2}$, and $C_{3}$ are arbitrary constants of integration.

Case 3. Substitution of the group-invariant solution corresponding to the subalgebra $X_{3}+a X_{2}$ into (2) results in the reduced nonlinear third-order $\mathrm{ODE}$

$$
(h-a) H^{\prime \prime \prime}-h^{\prime \prime}+2 h^{\prime} h^{\prime \prime}+a h^{\prime}+h=0,
$$

where "prime" denotes differentiation with respect to $\gamma$.

\section{Conservation Laws of (2)}

In this section, we obtain nonlocal conservation laws of (2) using the variational approach.

Equation (2) does not have a Lagrangian as it is an evolution equation. By making a substitution $u=v_{x}$, (2) becomes

$$
v_{x t}-2 v_{x x} v_{x x x}-v_{x} v_{x x x x}-v_{x x x t}=0
$$


Equation (24) has a usual Lagrangian

$$
L=-\frac{1}{2}\left(v_{t} v_{x}+v_{x} v_{x x}^{2}+v_{x t} v_{x x}\right)
$$

If $X=\tau \partial_{t}+\xi \partial_{x}+\eta \partial_{v}$ is the Noether symmetry operator, then from the Noether operator determining equation (8) we obtain

$$
\begin{gathered}
-\frac{1}{2} v_{x} \zeta_{t}-\frac{1}{2}\left(v_{t}+v_{x x}^{2}\right) \zeta_{x}-\left(v_{x} v_{x x}+\frac{1}{2} v_{x t}\right) \zeta_{x x} \\
-\frac{1}{2} v_{x x} \zeta_{x t}+\left(\tau_{t}+\tau_{v} v_{t}\right) L+\left(\xi_{x}+\xi_{v} v_{x}\right) L \\
=B_{t}^{1}+B_{v}^{1} v_{t}+B_{x}^{2}+B_{v}^{2} v_{x} .
\end{gathered}
$$

Expansion of (26) and then equating the coefficients of the various monomials in the first- and second-order partial derivatives of $v$ to zero yield the following determining equations:

$$
\begin{gathered}
\tau_{x}=\tau_{v}=0, \quad \xi_{t}=\xi_{v}=0, \\
\eta_{v}=0, \quad 4 \xi_{x}-\tau_{t}-3 \eta_{v}=0, \\
\eta_{v}-\xi_{x}, \quad \xi_{t}-\eta_{x}=0, \\
B_{v}^{1}=-\frac{1}{2} \eta_{x}, \quad B_{v}^{2}=-\frac{1}{2} \eta_{t}, \\
B_{t}^{1}+B_{x}^{2}=0 .
\end{gathered}
$$

The solution of (27) is $\tau=c_{1}, \xi=c_{2}, \eta=a(t), B^{1}=c(t, x)$, and $B^{2}=-1 / 2 a^{\prime}(t) v+d(t, x)$, where $c_{1}, c_{2}$ are arbitrary constants, $a(t)$ is an arbitrary function, and $c_{t}+d_{x}=0$. We set $c=d=0$. Thus, we obtain the following Noether symmetry operators associated with the Lagrangian (25) for (24):

$$
\begin{gathered}
X_{1}=\partial_{t}, \quad B^{1}=0, \quad B^{2}=0, \\
X_{2}=\partial_{x}, \quad B^{1}=0, \quad B^{2}=0, \\
X_{3}=a(t) \partial_{v}, \quad B^{1}=0, \quad B^{2}=-\frac{1}{2} a^{\prime}(t) v .
\end{gathered}
$$

Hence, by invoking (9), we obtain the following conserved vectors corresponding to the Noether symmetry operators (28):

(i) $X_{1}=\partial_{t}, B^{1}=0, B^{2}=0$,

$$
\begin{gathered}
T^{1}=\frac{1}{2} v_{x} v_{x x}^{2}+\frac{1}{2} v_{t} v_{x x x} \\
T^{2}=-\frac{1}{2} v_{t}^{2}+\frac{1}{2} v_{t} v_{x x}^{2}+v_{t} v_{x x t}+v_{t} v_{x} v_{x x x} \\
-\frac{1}{2} v_{t t} v_{x x}-v_{x} v_{t x} v_{x x}-\frac{1}{2} v_{t x}^{2}
\end{gathered}
$$

Hence, from (5), we have

$$
\begin{aligned}
D_{t}\left(T^{1}\right)+D_{x}\left(T^{2}\right)= & -v_{t}\left(v_{x t}-2 v_{x x} v_{x x x}-v_{x} v_{x x x x}-v_{x x x t}\right) \\
& +\frac{1}{2} v_{t} v_{x x x t}-\frac{1}{2} v_{x x} v_{x t t} \\
= & \frac{1}{2} v_{t} v_{x x x t}-\frac{1}{2} v_{x x} v_{x t t} \\
= & D_{t}\left(\frac{1}{2} v_{t} v_{x x x}\right)-D_{x}\left(\frac{1}{2} v_{x x} v_{t t}\right) .
\end{aligned}
$$

Taking these terms across and including them into the conserved flows, we get

$$
D_{t}\left(T^{1}-\frac{1}{2} v_{t} v_{x x x}\right)+D_{x}\left(T^{2}+\frac{1}{2} v_{x x} v_{t t}\right)=0 .
$$

If we let

$$
\begin{gathered}
\widetilde{T}^{1}=T^{1}-\frac{1}{2} v_{t} v_{x x x}=\frac{1}{2} v_{x} v_{x x}^{2}, \\
\widetilde{T}^{2}=T^{2}+\frac{1}{2} v_{x x} v_{t t} \\
=-\frac{1}{2} v_{t}^{2}+\frac{1}{2} v_{t} v_{x x}^{2}+v_{t} v_{x x t}+v_{t} v_{x} v_{x x x} \\
-v_{x} v_{t x} v_{x x}-\frac{1}{2} v_{t x}^{2},
\end{gathered}
$$

one can readily verify that the new components of the conserved vector $\widetilde{T}$ satisfy the equation $\left.D_{i} \widetilde{T}^{i}\right|_{(24)}=0$.

(ii) Consider $X_{2}=\partial_{x}, B^{1}=0, B^{2}=0$,

$$
\begin{aligned}
& T^{1}=-\frac{1}{2} v_{x}^{2}+\frac{1}{2} v_{x} v_{x x x}-\frac{1}{2} v_{x x}^{2}, \\
& T^{2}=v_{x} v_{x x t}+v_{x}^{2} v_{x x x}-\frac{1}{2} v_{x t} v_{x x} .
\end{aligned}
$$

Invoking (5), we obtain

$$
\begin{aligned}
D_{t}\left(T^{1}\right)+D_{x}\left(T^{2}\right)= & -v_{x}\left(v_{x t}-2 v_{x x} v_{x x x}-v_{x} v_{x x x x}-v_{x x x t}\right) \\
& +\frac{1}{2} v_{x} v_{x x x t}-\frac{1}{2} v_{x x} v_{x x t} \\
= & \frac{1}{2} v_{x} v_{x x x t}-\frac{1}{2} v_{x x} v_{x x t} \\
= & D_{x}\left(\frac{1}{2} v_{x} v_{x x t}\right)-D_{t}\left(\frac{1}{2} v_{x x}^{2}\right) .
\end{aligned}
$$

Taking the terms across and adding them into the conserved flows yield

$$
D_{t}\left(T^{1}+\frac{1}{2} v_{x x}^{2}\right)+D_{x}\left(T^{2}-\frac{1}{2} v_{x} v_{x x t}\right)=0 .
$$


Considering

$$
\begin{aligned}
\widetilde{T}^{1} & =T^{1}+\frac{1}{2} v_{x x}^{2}=\frac{1}{2} v_{x} v_{x x x}-\frac{1}{2} v_{x}^{2}, \\
\widetilde{T}^{2} & =T^{2}-\frac{1}{2} v_{x} v_{x x t} \\
& =\frac{1}{2} v_{x} v_{x x t}+v_{x}^{2} v_{x x x}-\frac{1}{2} v_{x t} v_{x x},
\end{aligned}
$$

we obtain the modified new components of the conserved vector $\widetilde{T}$ so that the equation, $\left.D_{i} \widetilde{T}^{i}\right|_{(24)}=0$, is satisfied.

(iii) Consider $X_{3}=a(t) \partial_{v}, B^{1}=0, B^{2}=-(1 / 2) a(t) v$,

$$
\begin{gathered}
T^{1}=\frac{1}{2} a(t) v_{x}-\frac{1}{2} a(t) v_{x x x}, \\
T^{2}=-\frac{1}{2} a^{\prime}(t) v+\frac{1}{2} a(t) v_{t}-\frac{1}{2} a(t) v_{x x}^{2} \\
-a(t) v_{x x t}-a(t) v_{x} v_{x x x} .
\end{gathered}
$$

In this case, we have infinite number of conservation laws. Using (5), we have

$$
\begin{aligned}
D_{t}\left(T^{1}\right)+D_{x}\left(T^{2}\right)= & a(t)\left(v_{x t}-2 v_{x x} v_{x x x}-v_{x} v_{x x x x}-v_{x x x t}\right) \\
& -\frac{1}{2}\left(a^{\prime}(t) v_{x x x}+a(t) v_{x x x t}\right) \\
= & -\frac{1}{2}\left(a^{\prime}(t) v_{x x x}+a(t) v_{x x x t}\right) \\
= & D_{t}\left(-\frac{1}{2} a(t) v_{x x x}\right) .
\end{aligned}
$$

Taking the term across and adding it into the conserved flows, we obtain

$$
D_{t}\left(T^{1}+\frac{1}{2} a(t) v_{x x x}\right)+D_{x}\left(T^{2}\right)=0 .
$$

If we choose

$$
\begin{gathered}
\widetilde{T}^{1}=T^{1}+\frac{1}{2} a(t) v_{x x x}=\frac{1}{2} a(t) v_{x}, \\
\widetilde{T}^{2}=T^{2},
\end{gathered}
$$

we obtain $\left.D_{i} \widetilde{T}^{i}\right|_{(24)}=0$.

\section{Concluding Remarks}

In this paper, we studied the modified Hunter-Saxton equation (2) using the Lie symmetry group of infinitesimal transformations of the equation. We found that the underlying equation admits a three-dimensional Lie algebra spanned by the vector fields of translations in time and space and the scaling of time and the dependent variable. We obtained the optimal system of one-dimensional subalgebras of the Lie algebra of the equation. These subalgebras were then used to reduce the underlying equation to nonlinear third-order ordinary differential equations. Exact group-invariant solutions called traveling wave solutions were constructed by integrating the reduced ODEs. Furthermore, we constructed infinite number of nonlocal conservation laws for the underlying equation by the transformation of the dependent variable of the equation and making use of Noether's theorem.

\section{References}

[1] J. K. Hunter and R. Saxton, "Dynamics of director fields," SIAM Journal on Applied Mathematics, vol. 51, no. 6, pp. 1498-1521, 1991.

[2] H.-H. Dai and M. Pavlov, "Transformations for the CamassaHolm equation, its high-frequency limit and the Sinh-Gordon equation," Journal of the Physical Society of Japan, vol. 67, no. 11, pp. 3655-3657, 1998.

[3] J. K. Hunter and Y. X. Zheng, "On a completely integrable nonlinear hyperbolic variational equation," Physica D, vol. 79, no. 2-4, pp. 361-386, 1994.

[4] B. Khesin and G. Misiołek, "Euler equations on homogeneous spaces and Virasoro orbits," Advances in Mathematics, vol. 176, no. 1, pp. 116-144, 2003.

[5] J. Lenells, "The Hunter-Saxton equation: a geometric approach," SIAM Journal on Mathematical Analysis, vol. 40, no. 1, pp. 266277, 2008.

[6] V. A. Galaktionov and S. R. Svirshchevskii, Exact Solutions and Invariant Subspaces of Nonlinear Partial Differential Equations in Mechanics and Physics, Chapman \& Hall/CRC, New York, NY, USA, 2007.

[7] N. H. Ibragimov, A. H. Kara, and F. M. Mahomed, "LieBäcklund and Noether symmetries with applications," Nonlinear Dynamics, vol. 15, no. 2, pp. 115-136, 1998.

[8] A. H. Kara and F. M. Mahomed, "Relationship between symmetries and conservation laws," International Journal of Theoretical Physics, vol. 39, no. 1, pp. 23-40, 2000.

[9] E. Noether, "Invariant variation problems," Transport Theory and Statistical Physics, vol. 1, no. 3, pp. 186-207, 1971.

[10] P. J. Olver, Applications of Lie Groups to Differential Equations, Springer, New York, NY, USA, 2nd edition, 1993.

[11] G. W. Bluman and S. Kumei, Symmetries and Differential Equations, vol. 81, Springer, New York, NY, USA, 1989.

[12] N. H. Ibragimov, CRC Handbook of Lie Group Analysis of Differential Equations, vol. 1-3, CRC Press, Boca Raton, Fla, USA, 1994.

[13] L. V. Ovsiannikov, Group Analysis of Differential Equations, Academic Press, New York, NY, USA, 1982.

[14] H. Liu and J. Li, "Lie symmetries, conservation laws and exact solutions for two rod equations," Acta Applicandae Mathematicae, vol. 110, no. 2, pp. 573-587, 2010. 


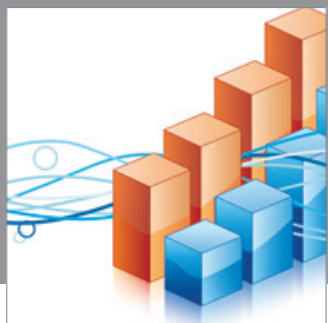

Advances in

Operations Research

mansans

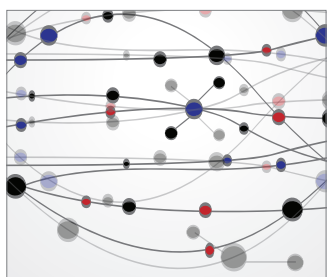

The Scientific World Journal
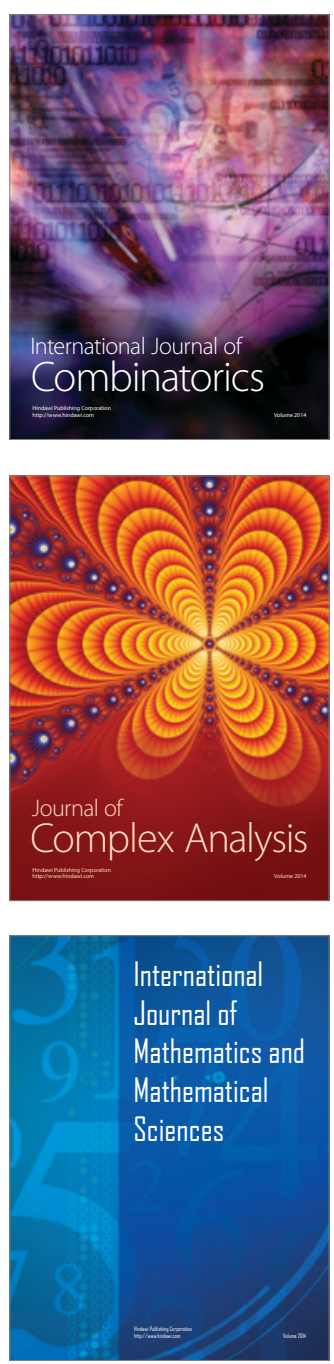
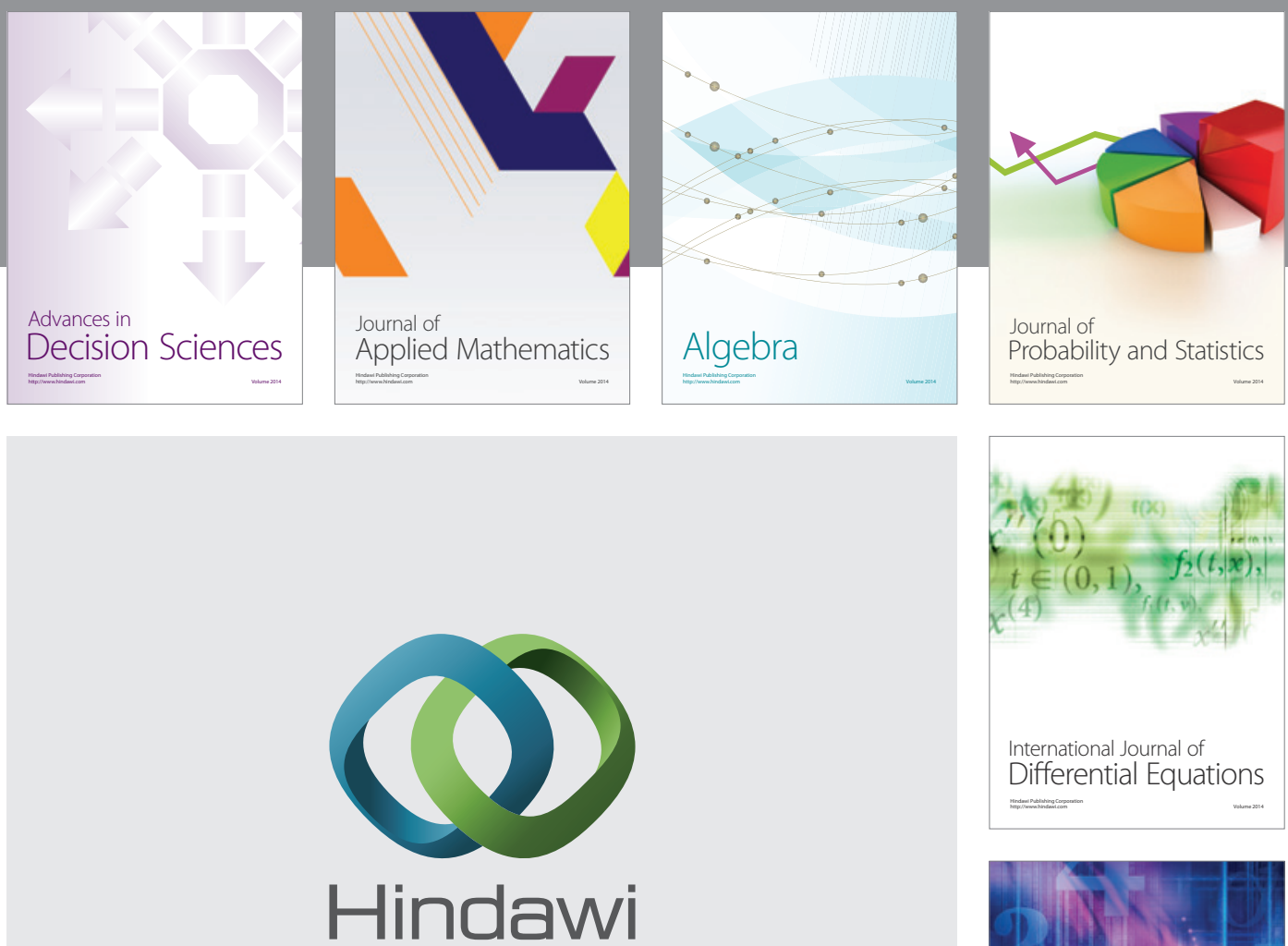

Submit your manuscripts at http://www.hindawi.com
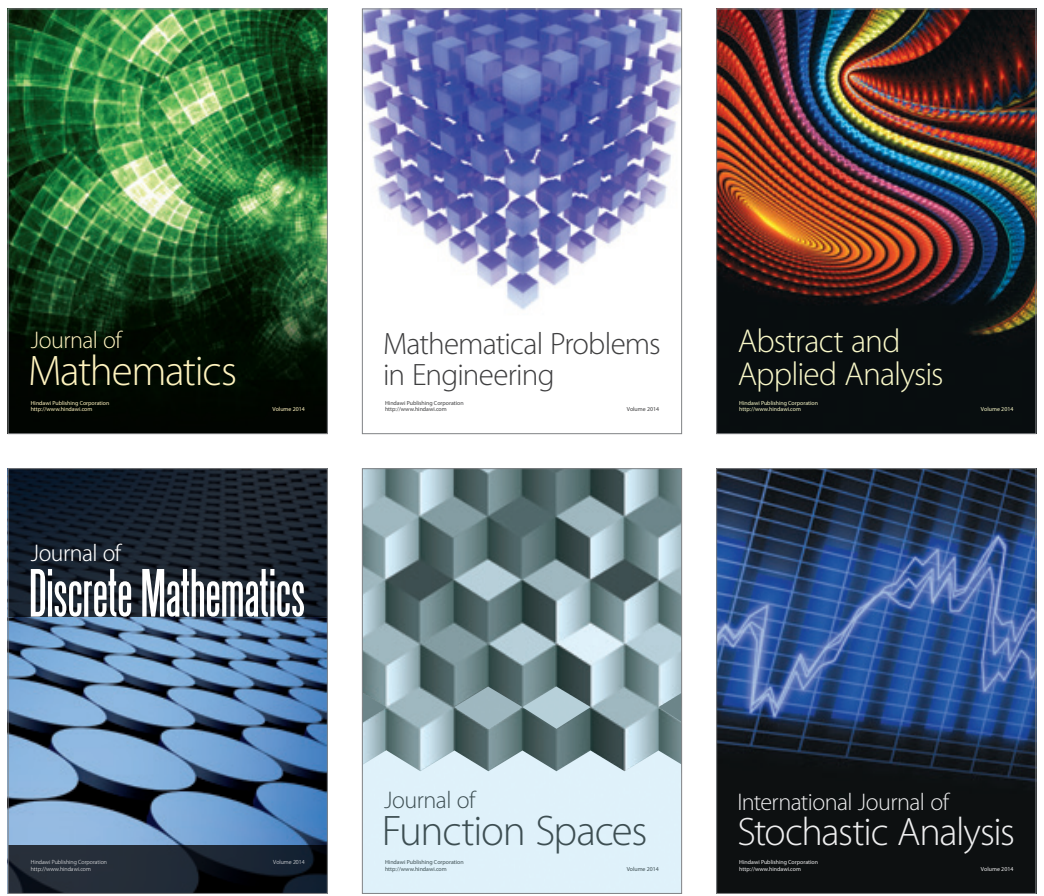

Journal of

Function Spaces

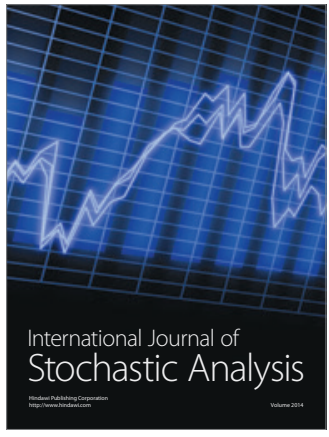

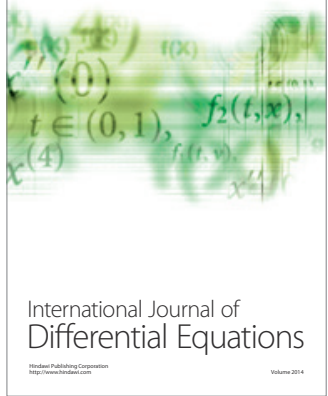
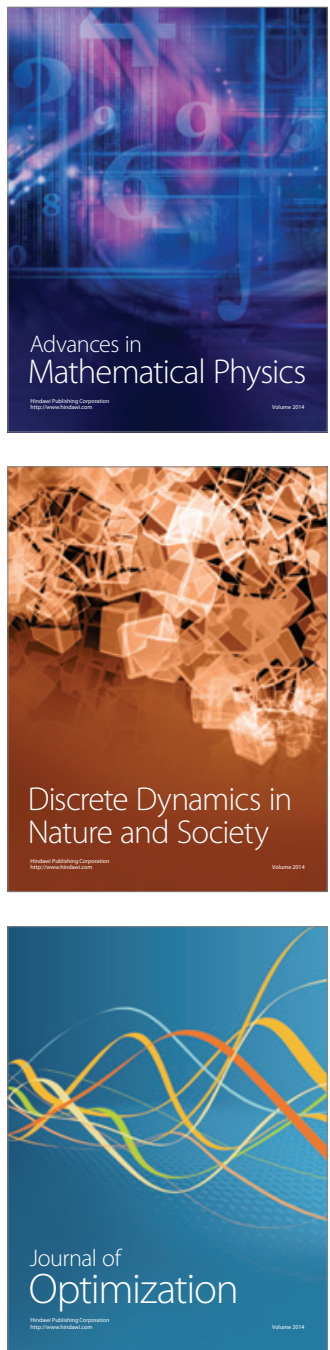